\title{
Measurement of Intraoperative Graft Flow Predicts Radiological Hyperperfusion during Bypass Surgery in Patients with Moyamoya Disease
}

\author{
Akikazu Nakamura ${ }^{a}$ Akitsugu Kawashimaa Shunsuke Nomura ${ }^{a}$ \\ Takakazu Kawamatab \\ ${ }^{a}$ Department of Neurosurgery, Tokyo Women's Medical University Yachiyo Medical Center,

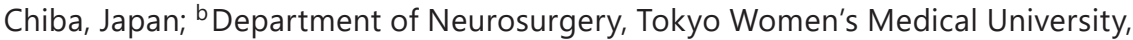 \\ Tokyo, Japan
}

Keywords

Bypass surgery $\cdot$ Hyperperfusion · Moyamoya disease $\cdot$ Transit time flowmeter

\begin{abstract}
Introduction: Moyamoya disease (MMD) is a rare cerebrovascular disease associated with cerebral infarction or hemorrhage. Hyperperfusion is the most significant complication of direct bypass surgery. Previous research has shown that an increase in cerebral blood flow (CBF) is strongly related to symptomatic hyperperfusion and highlighted the importance of postoperative assessment of CBF. Objective: The principal aims of this study were to quantitatively analyze the relationship between intraoperative graft flow and increase in CBF and to evaluate the effectiveness of intraoperative graft flow measurement during bypass surgery for patients with MMD. Methods: This study included 91 surgeries in 67 consecutive adult patients with MMD who underwent direct revascularization surgery at our institution between November 2013 and September 2018. Intraoperative graft flow of the branches and main trunk was measured in all patients, after anastomosis had been established. Postoperative CBF measurements were performed under sedation, immediately after surgery. Radiological hyperperfusion was defined as focal high uptake, as determined by CBF imaging immediately after surgery. Patients were divided into two groups (radiological hyperperfusion and nonradiological hyperperfusion groups), and the relationship between intraoperative graft flow and radiological hyperperfusion was analyzed. Results: Significant differences were observed between the radiological hyperperfusion and nonradiological hyperperfusion groups in terms of intraoperative graft flow of both the branch (median 72 vs. $42 \mathrm{~mL} / \mathrm{min}$, respectively; $p<$ 0.01 ) and main trunk (median 113 vs. $68 \mathrm{~mL} / \mathrm{min}$, respectively; $p<0.01$ ). A receiver-operating characteristic analysis was performed to test the utility of intraoperative flow as a quantitative
\end{abstract}


measure. We set the cutoff values for the intraoperative branch and main trunk flow at $57 \mathrm{~mL} /$ min (sensitivity: 0.707, specificity: 0.702; area under the curve [AUC]: 0.773; 95\% confidence interval [Cl]: $0.675-0.871$ ) and $84 \mathrm{~mL} / \mathrm{min}$ (sensitivity: 0.667 , specificity: 0.771 ; AUC: $0.78 ; 95 \%$ $\mathrm{Cl}$ : $0.685-0.875)$, respectively. Conclusions: Measuring intraoperative graft flow during bypass surgery may be an effective means of predicting hyperperfusion and could serve to facilitate early therapeutic intervention such as strict blood pressure control.

(C) 2020 The Author(s)

Published by S. Karger AG, Basel

\section{Introduction}

Moyamoya disease (MMD) is a rare cerebrovascular disease characterized by progressive occlusion of the terminal internal carotid artery and the formation of abnormal moyamoya vessels, which can lead to cerebral infarction or hemorrhage [1, 2]. Several studies have demonstrated the effectiveness of using direct or combined bypass surgery [3-7].

Hyperperfusion syndrome is the most significant complication of direct bypass surgery and can lead to temporary neurological deterioration or hemorrhagic stroke [8-11]. Previous research has shown that an increase in cerebral blood flow (CBF) is strongly related to symptomatic hyperperfusion and highlighted the importance of postoperative CBF studies $[11,12]$.

Intraoperative flow measurement of the extracranial-intracranial bypass graft was considered to be a simple technique for predicting cerebral hyperperfusion during direct bypass surgery in previous reports $[13,14]$. However, to date, no studies have performed a quantitative analysis of the relationship between intraoperative graft flow and cerebral hyperperfusion during bypass surgery.

Therefore, the principal goal of this study was to quantitatively analyze this relationship and to evaluate the effectiveness of intraoperative graft flow measurement in bypass surgery for MMD.

\section{Materials and Methods}

This retrospective study was approved by the Institutional Review Board of Tokyo Women's Medical University.

\section{Patient Population and Surgical Procedures}

This study analyzed 91 surgeries in 67 consecutive adult patients with MMD (mean age: 39 years, range: 16-75 years), who underwent direct revascularization with concurrent measurement of intraoperative graft flow at our institution between November 2013 and September 2018. All patients were diagnosed according to criteria stipulated by the Japanese Ministry of Health, Labor, and Welfare [15]. No patients were diagnosed as quasi-MMD in this population, due to the strict application of these criteria. Our surgical indications were as follows: ischemic symptoms and decreased CBF, or progressive ischemic symptoms. The following direct bypass procedures were included in the study: 77 superficial temporal artery (STA)-middle cerebral artery (MCA) single or double bypasses, 2 STA-anterior cerebral artery and MCA double bypasses, 4 retroauricular artery and STA-MCA double bypasses, and 8 occipital artery-MCA single or double bypasses. A double bypass was performed in 79 out of $91(87 \%)$ surgeries. In our institution, the STA-MCA double bypass was the standard surgical procedure. In cases where low CBF was detected in the anterior cerebral artery area, we performed an STA-anterior cerebral artery bypass. In cases where the appropriate STA was

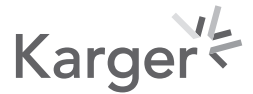




\section{Cerebrovascular \\ Diseases Extra}
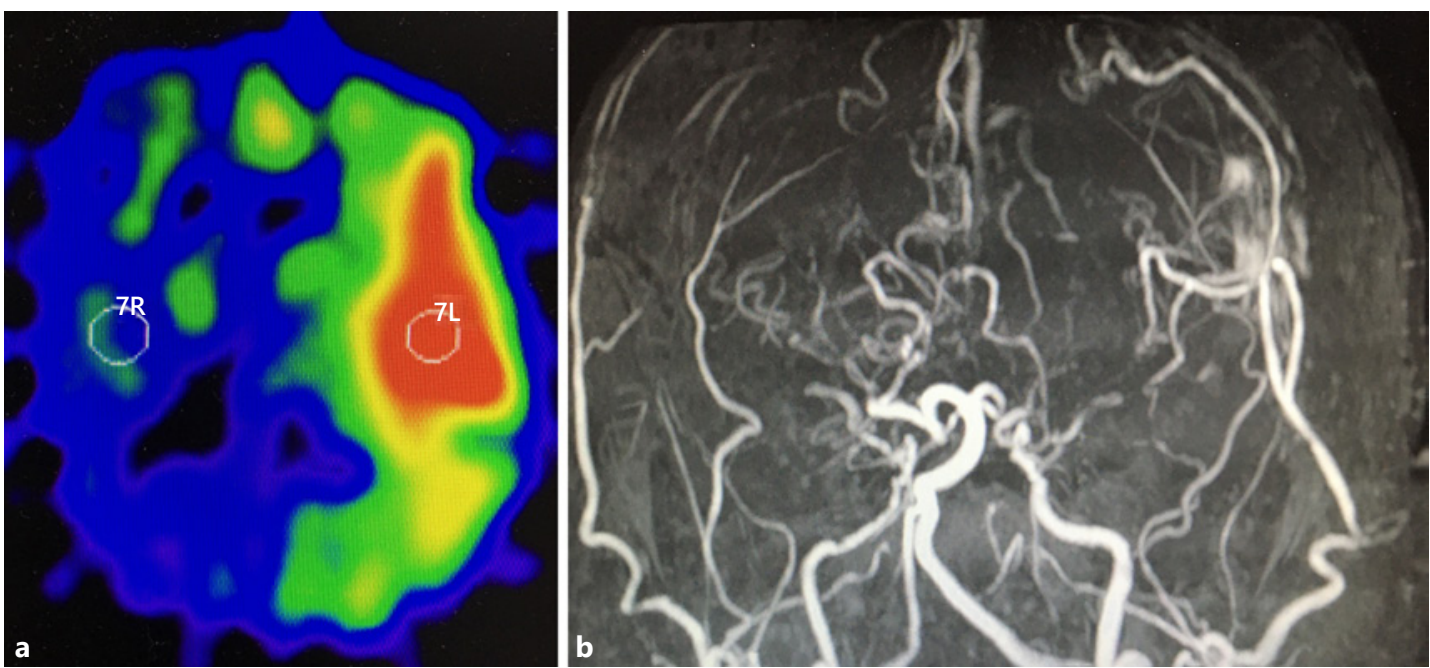

Fig. 1. a Analysis of single-photon emission computed tomography immediately after surgery. b Example of magnetic resonance angiography after surgery.

not available, we performed a retroauricular artery and STA-to-MCA double bypass. In cases that needed additional revascularization after the initial one, we performed an occipital artery-MCA bypass.

\section{Preoperative Radiological Examination}

All patients underwent magnetic resonance imaging (MRI), MR angiography, and conventional angiography prior to surgery. The disease was classified into 6 stages, according to Suzuki's angiographic staging. CBF and cerebrovascular reserve (CVR) were measured preoperatively in all patients using [ $\left.{ }^{123} \mathrm{I}\right] \mathrm{N}$-isopropyl-p-iodo-amphetamine ( ${ }^{123} \mathrm{I}$-IMP) singlephoton emission computed tomography (SPECT).

\section{Intraoperative Graft Flow of the Branches and Main Trunk}

Intraoperative graft flow of the branches and main trunk was measured in each patient $10 \mathrm{~min}$ after establishing the anastomosis in the double-bypass procedure in a single simultaneous measurement. The total flow of the frontal and parietal branches was used as an estimate if the bifurcation of the graft could not be visualized intraoperatively and the main trunk flow could not be measured. We measured blood flow using a flexible perivascular flow probe (H2MB - $2 \mathrm{~mm}, \mathrm{H} 3 \mathrm{MB}-3 \mathrm{~mm}$; Transonic Systems Inc., Ithaca, NY, USA) and dual channel flow meter (HT207; Transonic Systems Inc., Ithaca, NY, USA) equipped with a personal computer interface for waveform analysis (Chart for Windows 3.3.5, ADInstruments, Sydney, Australia), based on an earlier study [14].

\section{Postoperative Management}

Postoperative CBF measurements were performed under sedation, immediately after surgery. We defined radiological hyperperfusion (RHP) as focal high uptake detected by ${ }^{123}$ I-IMP SPECT immediately after revascularization surgery, as per previous studies $[8,16]$. In cases that were difficult to evaluate, RHP was defined as an increase in CBF of more than $200 \%$ relative to that of the ipsilateral cerebellum, represented by a $1-\mathrm{cm}$ diameter circle in areas that commonly show high uptake (Fig. 1a). RHP was independently diagnosed by two neurosurgeons. In all patients, blood pressure (BP) was maintained under $140 \mathrm{~mm} \mathrm{Hg}$, and they were placed under sedation until postoperative CBF measurement was finished. 
Table 1. Characteristics of patients with radiological versus nonradiological hyperperfusion

\begin{tabular}{lccc}
\hline & $\begin{array}{l}\text { RHP } \\
(n=49 ; 54 \%)\end{array}$ & $\begin{array}{l}\text { Control } \\
(n=42 ; 46 \%)\end{array}$ & $p$ value \\
\hline Median age [first and third quartiles], years & $43[36,53]$ & $38[33,44]$ & 0.0544 \\
Females & $33(67 \%)$ & $27(64 \%)$ & 0.662 \\
Left-sided surgery & $22(45 \%)$ & $21(50 \%)$ & 0.834 \\
Hypertension & $13(27 \%)$ & $7(17 \%)$ & 0.311 \\
Diabetes mellitus & $3(6 \%)$ & $1(2 \%)$ & 0.62 \\
Dyslipidemia & $3(6 \%)$ & $0(0 \%)$ & 0.245 \\
Suzuki grade $>4$ & $24(49 \%)$ & $13(31 \%)$ & 0.0842 \\
Clinical presentation & $2(4 \%)$ & $1(2 \%)$ & 0.603 \\
$\quad$ Hemorrhage & $11(22 \%)$ & $10(24 \%)$ & $26(62 \%)$ \\
$\quad$ Infarction & $22(45 \%)$ & $1(2 \%)$ & \\
$\quad$ TIA & $0(0 \%)$ & $5(12 \%)$ & 0.583 \\
$\quad$ Headache & $7(14 \%)$ & $2(5 \%)$ & \\
$\quad$ Osymptomatic & $6(12 \%)$ & $6(14 \%)$ & \\
Double anastomosis of the supra-Sylvian artery & $10(20 \%)$ & & \\
\hline
\end{tabular}

RHP, radiological hyperperfusion; TIA, transient ischemic attack. Numbers represent total numbers of participants meeting the specific criteria, unless otherwise specified.

In patients with RHP, BP was maintained under $120 \mathrm{~mm}$ Hg until postoperative day 7. Patients with RHP were placed under sedation with propofol, dexmedetomidine hydrochloride, or thiopental sodium depending on the patient's condition. Carbon dioxide $\left(\mathrm{CO}_{2}\right)$ was maintained at approximately $40 \mathrm{~mm} \mathrm{Hg}$ during the sedation period. MRI was performed to check for patency of the graft flow during the first week after surgery (Fig. 1b). In patients with no RHP, BP was maintained under $140 \mathrm{~mm} \mathrm{Hg}$ until postoperative day 7. They were not placed under sedation.

\section{Data Analysis}

We divided patients into two groups: an RHP and a control group. The RHP group included patients who had RHP, and the control group included all patients without RHP. The following factors were then analyzed for each group: age; proportion of women; proportion of leftsided surgeries; history of hypertension, diabetes mellitus, and dyslipidemia; proportion of patients with Suzuki grade $>4$ MMD; proportion of patients with a clinical presentation suggestive of cerebral hemorrhage, cerebral infarction, or transient ischemic attack, as well as those presenting with headache and those asymptomatic; preoperative ipsilateral and contralateral CBF; preoperative ipsilateral CVR; presence of a double anastomosis to the supra-Sylvian artery; intraoperative graft flow of the branch with the higher flow and main trunk; and $\mathrm{CO}_{2}$ as well as mean $\mathrm{BP}$ during intraoperative graft flow measurement.

\section{Statistical Analyses}

All statistical analyses were performed using R software (The R Foundation for Statistical Computing, Vienna, Austria). $p$ values $<0.05$ were considered statistically significant. The Fisher exact test and the Mann-Whitney U test were used to compare differences between the two groups. Univariate and multivariate receiver-operating characteristic (ROC) analyses were also performed. For multivariate analysis, we incorporated factors known to influence hyperperfusion $[8,12,17,18]$. We defined the cutoff value as the point closest to the upper left side of the ROC graph. 
Table 2. Results of the cerebral blood flow analysis and intraoperative graft flow measurements

\begin{tabular}{llrr}
\hline & $\begin{array}{l}\text { RHP } \\
(n=49)\end{array}$ & $\begin{array}{l}\text { Control } \\
(n=42)\end{array}$ & $\begin{array}{l}p \\
\text { value }\end{array}$ \\
\hline Ipsilateral CBF, $\mathrm{mL} / 100 \mathrm{~g} / \mathrm{min}$ & $43.24(37.05,47.37)$ & $44.84(39.46,50.36)$ & 0.268 \\
Contralateral CBF, $\mathrm{mL} / 100 \mathrm{~g} / \mathrm{min}$ & $44.98(40.05,49.99)$ & $46.08(40.72,49.82)$ & 0.851 \\
Ipsilateral CVR, \% & $19.87(11.45,30.28)$ & $21.20(12.28,40.02)$ & 0.388 \\
Intraoperative flow branch, $\mathrm{mL} / \mathrm{min}$ & $72(49,87)$ & $42(32,60)$ & $<0.001$ \\
Intraoperative flow main trunk, $\mathrm{mL} / \mathrm{min}$ & $113(85,130)$ & $68(53,96)$ & $<0.001$ \\
$\mathrm{CO}_{2}$, mm Hg & $38(35,41)$ & $38(36,40)$ & 0.713 \\
Blood pressure, mm Hg & $69(63,77)$ & $69(65,74)$ & 0.885 \\
\hline
\end{tabular}

Results are presented as medians with first and third quartiles in parentheses. CBF, cerebral blood flow; CVR, cerebrovascular reserve; RHP, radiological hyperperfusion.

\section{Results}

RHP occurred in association with 49 of 91 surgeries (54\%) and symptomatic hyperperfusion with 38 of 91 surgeries (42\%). There were no significant differences in patient characteristics and preoperative CBF study results between the RHP and non-RHP groups (Tables 1 and 2). There were significant differences between the RHP and non-RHP groups in the intraoperative graft flow of the branch (median 72 vs. $42 \mathrm{~mL} / \mathrm{min}$, respectively; $p<0.001$ ) and the main trunk (median 113 vs. $68 \mathrm{~mL} / \mathrm{min}$, respectively; $p<0.001$ ).

ROC analysis was performed in order to evaluate intraoperative flow as a quantitative measure. We set the cutoff value at $57 \mathrm{~mL} / \mathrm{min}$ for intraoperative graft flow of the branch (sensitivity: 0.707; specificity: 0.702; area under the curve [AUC]: 0.773; 95\% confidence interval: $0.675-0.871$ ) and at $84 \mathrm{~mL} / \mathrm{min}$ for the main trunk (sensitivity: 0.667 ; specificity: 0.771; AUC: 0.78 ; $95 \%$ confidence interval: $0.685-0.875$; Fig. 1a, b). The $95 \%$ sensitivity value was $35 \mathrm{~mL} / \mathrm{min}$ on intraoperative graft flow of the branch and $40 \mathrm{~mL} / \mathrm{min}$ on the main trunk. The $95 \%$ specificity value was $71 \mathrm{~mL} / \mathrm{min}$ on intraoperative graft flow of the branch and 118 $\mathrm{mL} / \mathrm{min}$ on the main trunk. Multivariate ROC analysis revealed that intraoperative flow of the branch (AUC: 0.818 [branch] vs. 0.613 [control], $p<0.001$ ) and main trunk (AUC: 0.824 [main trunk] vs. 0.616 [control], $p<0.001$ ) was an independent factor for predicting RHP (Fig. 2c, d).

The patients were divided into 2 groups, based on whether the intraoperative graft flow of the branch and the main trunk were beyond the cutoff value or not, and analyzed the relation between these groups and RHP. Significant differences were found when compared to RHP: intraoperative graft flow of the branch (66 vs. $29 \% ; p<0.001$ ) and main trunk (77 vs. $33 \% ; p<0.001)$.

\section{Discussion}

\section{Intraoperative Graft Flow Measurement}

This is the first study to demonstrate a significant correlation between intraoperative graft flow and hyperperfusion during bypass surgery in patients with MMD. The ROC analysis revealed that intraoperative flow can predict hyperperfusion quantitatively, and multivariate ROC analysis proved the statistical rationale of this study. We set the cutoff value as the point closest to the upper left side of the ROC graph to achieve a balance between sensitivity and specificity. If the graft flow exceeds this cutoff value, the hyperperfusion can be better managed

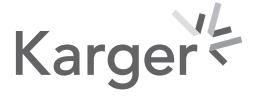



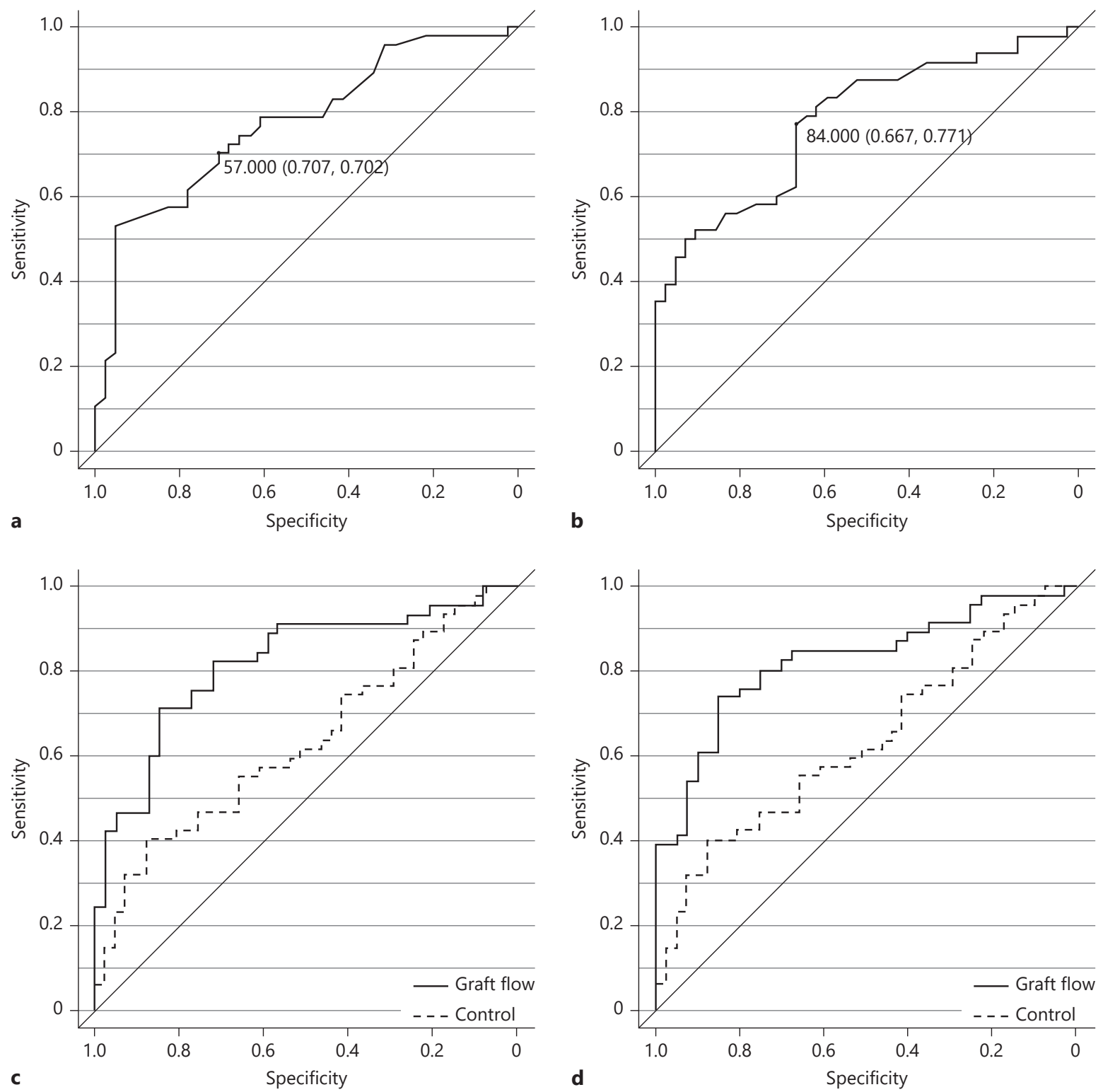

Fig. 2. a, b Univariate receiver-operating characteristic (ROC) analysis of intraoperative graft flow of the branch (a) and the main trunk (b). The cutoff value was set at $57 \mathrm{~mL} / \mathrm{min}$ (sensitivity: 0.707, specificity: 0.702 ) and $84 \mathrm{~mL} / \mathrm{min}$ (sensitivity: 0.667, specificity: 0.771), respectively. c, d Multivariate ROC analysis of intraoperative graft flow of the branch (c; area under the curve, AUC: 0.818 vs. $0.613, p=0.001$ ) and the main trunk (d; AUC: 0.824 vs. $0.616 p=0.001$ ), respectively. The factors included in the multivariate analysis: age; rates of left-sided surgery; hemorrhagic presentation; Suzuki grade $>4$; and preoperative ipsilateral cerebrovascular reserve as a risk factor for hyperperfusion.

through the implementation of strict BP control and sedation in the immediate postoperative period. There is an advantage of intraoperative graft flow measurement over SPECT. Although patients in our institution can undergo SPECT immediately postoperatively, in most other centers this is not the case due to preparation of nuclide. Our study has shown that intraoperative graft flow can provide early and easy prediction of RHP. 
Several studies have demonstrated the effectiveness of intraoperative graft flow measurement for predicting complications after revascularization in patients with MMD. One study with a mixed cohort of patients with internal carotid artery occlusion and MMD showed that postoperative hyperperfusion occurred in cases where STA flow was higher than $50 \mathrm{~mL} /$ $\min [14]$. However, this was true only for patients with internal carotid artery occlusion; none of the 7 patients with MMD demonstrated postoperative hyperperfusion. Therefore, the small sample size may not have been sufficient to quantitatively prove the effectiveness of intraoperative graft flow measurement in patients with MMD. In another study with a large sample size, a relationship between increased intraoperative graft flow and postoperative stroke, including hemorrhage, was reported [19]. However, the relationship between intraoperative graft flow and other cerebral hemodynamic parameters, as measured by xenon-enhanced CT and SPECT, remained unclear in that study. The authors also indicated that it was not possible to directly compare the individual flow rates of a given vessel with regional blood flow. Moreover, they only reported the average intraoperative graft flow in patients with complications and did not use a cutoff value as a quantitative means for predicting complications. The present study demonstrated that a cutoff intraoperative flow value can be used to quantitatively predict hyperperfusion. We performed SPECT under sedation, immediately after surgery. This means that the effects of anesthesia were excluded from our results, because both the intraoperative graft flow measurement and SPECT were carried out under anesthesia or sedation.

\section{Hyperperfusion}

A definition of RHP has been provided in previous reports $[8,11,16,17,20]$, and our own definition of RHP is stated in the Materials and Methods section. Hyperperfusion was not defined as the average CBF in the MCA territory, but rather as the focal increase in CBF, since most complications of bypass surgery occur focally. Some reports also define RHP as focal high uptake $[8,16,17]$. Sedation has the possibility of decreasing CBF. It can also cause falsenegative results for RHP. However, our study showed an RHP rate of $54 \%$, which is consistent with a previous study that showed a 50\% rate [12]. This suggests that our CBF measurement can be considered reliable.

In our previous study, CVR was considered to be a predictor of delayed hyperperfusion [18]. However, in this study, there was no significant difference between RHP and preoperative CVR. This study included both delayed and early hyperperfusion. Another previous report had shown that CVR was not a predictor of hyperperfusion [12]. As the results of this present study suggest that preoperative CVR is not such a predictor, it is possible that other patient characteristics might have affected these results. Further studies that control for more parameters may provide a more reliable answer.

We evaluated RHP using SPECT immediately after surgery in order to predict RHP. One report showed that 5 out of 6 patients (83\%) had postoperative hemorrhage during postoperative days $0-1$, and 2 out of 6 patients (33\%) had postoperative hemorrhage prior to SPECT assessment [10]. Thus, intraoperative graft flow measurement can serve as a means for evaluating RHP intraoperatively. This ensures that patients with hyperperfusion will remain under sedation and undergo strict BP control after surgery. The importance of postoperative BP control [10,21] and sedation [18] after revascularization for MMD have been mentioned in previous reports. In addition, we consider sedation to make strict BP control easier. In our institution, BP was maintained below $120 \mathrm{~mm} \mathrm{Hg}$ and, whilst there is a theoretical risk of ischemic complications due to low BP, in this cohort no patients had ischemic complications during the postoperative period.

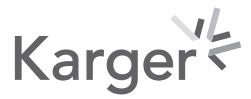




\section{Limitations}

There are several limitations to our study. First, the study was of retrospective design and conducted in a single center, which may have been affected by some degree of selection bias. Second, most of our patients underwent double-bypass surgery. However, in some institutions, patients with MMD undergo single-bypass surgery. The effectiveness of intraoperative graft flow measurements during single-bypass surgery is unknown and should be the subject of further study. However, it should be noted that we analyzed intraoperative graft flow of individual branches thought to be responsible for RHP, and it is therefore possible that the results of this particular analysis may be applicable to single-bypass cases. Third, while this study demonstrated a relationship between intraoperative graft flow and RHP, other factors may also play a role. It is interesting to note that some patients had RHP despite low intraoperative graft flow, and vasospasm of the graft during the surgical procedure is considered to be one of the causes of this phenomenon. Fourth, our study focused on radiographic and not symptomatic hyperperfusion in detail as an outcome measure, and RHP does not always translate into symptomatic hyperperfusion. However, we feel that it is important to be able to predict and manage RHP in order to prevent the development of symptomatic hyperperfusion. Finally, our study was performed in a single center, and a multicenter study is required to evaluate the optimal cutoff values for intraoperative graft flow, because surgical techniques may differ among institutions.

\section{Conclusion}

Our study demonstrated a significant correlation between intraoperative graft flow and cerebral hyperperfusion. In particular, the measurement of intraoperative graft flow during bypass surgery for MMD may be an effective means of predicting postoperative hyperperfusion. Intraoperative prediction of hyperperfusion could lead to faster implementation of therapeutic interventions such as strict BP control. A larger, multicenter study is required in order to evaluate in further detail the optimal cutoff values for intraoperative graft flow.

\section{Statement of Ethics}

The patients gave informed consent for inclusion in this study. This retrospective study was approved by the Institutional Review Board of Tokyo Women's Medical University.

\section{Disclosure Statement}

The authors have no conflicts of interest to declare.

\section{Funding Sources}

This study did not receive any funding. 


\section{Cerebrovascular Diseases Extra}

\section{Author Contributions}

Conception and design: Nakamura, Kawashima, Nomura. Acquisition of data: Nakamura, Kawashima, Kawamata. Analysis and interpretation of data: Nakamura, Kawashima, Nomura. Drafting the article: Nakamura, Kawashima, Nomura. Critically revising the article: Nakamura, Kawashima, Nomura, Kawamata. Reviewed submitted version of paper: Nakamura, Kawashima, Nomura, Kawamata. Statistical analysis: Nakamura. Administrative/technical/material support: Kawashima, Kawamata. Study supervision: Kawashima, Nomura, Kawamata.

\section{References}

1 Kuroda S, Houkin K. Moyamoya disease: current concepts and future perspectives. Lancet Neurol. 2008 Nov; $7(11): 1056-66$.

2 Uchino H, Kim JH, Fujima N, Kazumata K, Ito M, Nakayama N, et al. Synergistic interactions between direct and indirect bypasses in combined procedures: the significance of indirect bypasses in moyamoya disease. Neurosurgery. 2017 Feb;80(2):201-9.

3 Miyamoto S, Yoshimoto T, Hashimoto N, Okada Y, Tsuji I, Tominaga T, et al.; JAM Trial Investigators. Effects of extracranial-intracranial bypass for patients with hemorrhagic moyamoya disease: results of the Japan Adult Moyamoya Trial. Stroke. 2014 May;45(5):1415-21.

4 Ishikawa T, Houkin K, Kamiyama H, Abe H. Effects of surgical revascularization on outcome of patients with pediatric moyamoya disease. Stroke. 1997 Jun;28(6):1170-3.

5 Karasawa J, Touho H, Ohnishi H, Miyamoto S, Kikuchi H. Long-term follow-up study after extracranial-intracranial bypass surgery for anterior circulation ischemia in childhood moyamoya disease. J Neurosurg. 1992 Jul; 77(1):84-9.

6 Houkin K, Ishikawa T, Yoshimoto T, Abe H. Direct and indirect revascularization for moyamoya disease surgical techniques and peri-operative complications. Clin Neurol Neurosurg. 1997 Oct;99 Suppl 2:S142-5.

7 Miyamoto S, Akiyama Y, Nagata I, Karasawa J, Nozaki K, Hashimoto N, et al. Long-term outcome after STA-MCA anastomosis for moyamoya disease. Neurosurg Focus. 1998 Nov;5(5):e5.

8 Fujimura M, Mugikura S, Kaneta T, Shimizu H, Tominaga T. Incidence and risk factors for symptomatic cerebral hyperperfusion after superficial temporal artery-middle cerebral artery anastomosis in patients with moyamoya disease. Surg Neurol. 2009 Apr;71(4):442-7.

9 Yu J, Shi L, Guo Y, Xu B, Xu K. Progress on complications of direct bypass for moyamoya disease. Int J Med Sci. 2016 Jul;13(8):578-87.

10 Tokairin K, Kazumata K, Uchino H, Ito M, Ono K, Tatezawa R, et al. Postoperative intracerebral hemorrhage after combined revascularization surgery in moyamoya disease: profiles and clinical associations. World Neurosurg. 2018 Dec;120:e593-600.

11 Ishikawa T, Yamaguchi K, Kawashima A, Funatsu T, Eguchi S, Matsuoka G, et al. Predicting the occurrence of hemorrhagic cerebral hyperperfusion syndrome using regional cerebral blood flow after direct bypass surgery in patients with moyamoya disease. World Neurosurg. 2018 Nov;119:e750-6.

12 Uchino H, Kuroda S, Hirata K, Shiga T, Houkin K, Tamaki N. Predictors and clinical features of postoperative hyperperfusion after surgical revascularization for moyamoya disease: a serial single photon emission CT/ positron emission tomography study. Stroke. 2012 Oct;43(10):2610-6.

13 Lee M, Zaharchuk G, Guzman R, Achrol A, Bell-Stephens T, Steinberg GK. Quantitative hemodynamic studies in moyamoya disease: a review. Neurosurg Focus. 2009 Apr;26(4):E5.

14 Nakayama N, Kuroda S, Houkin K, Takikawa S, Abe H. Intraoperative measurement of arterial blood flow using a transit time flowmeter: monitoring of hemodynamic changes during cerebrovascular surgery. Acta Neurochir (Wien). 2001;143(1):17-24.

15 Hashimoto N, Tominaga T, Miyamoto S, Nagata I, Houkin K, Suzuki N, et al.; Health Labour Sciences Research Grant for Research on Measures for Infractable Diseases. Guidelines for diagnosis and treatment of moyamoya disease (spontaneous occlusion of the circle of Willis). Neurol Med Chir (Tokyo). 2012;52(5):245-66.

16 Fujimura M, Tominaga T. Significance of cerebral blood flow analysis in the acute stage after revascularization surgery for moyamoya disease. Neurol Med Chir (Tokyo). 2015;55(10):775-81.

17 Fujimura M, Niizuma K, Inoue T, Sato K, Endo H, Shimizu H, et al. Minocycline prevents focal neurological deterioration due to cerebral hyperperfusion after extracranial-intracranial bypass for moyamoya disease. Neurosurgery. 2014 Feb;74(2):163-70.

18 Nomura S, Yamaguchi K, Ishikawa T, Kawashima A, Okada Y, Kawamata T. Factors of delayed hyperperfusion and the importance of repeated cerebral blood flow evaluation for hyperperfusion after direct bypass for moyamoya disease. World Neurosurg. 2018 0ct;118:e468-72.

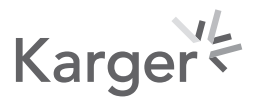


19 Lee M, Guzman R, Bell-Stephens T, Steinberg GK. Intraoperative blood flow analysis of direct revascularization procedures in patients with moyamoya disease. J Cereb Blood Flow Metab. 2011 Jan;31(1):262-74.

20 Kuroda S, Kamiyama H, Abe H, Asaoka K, Mitsumori K. Temporary neurological deterioration caused by hyperperfusion after extracranial-intracranial bypass-case report and study of cerebral hemodynamics. Neurol Med Chir (Tokyo). 1994 Jan;34(1):15-9.

21 Fujimura M, Inoue T, Shimizu H, Saito A, Mugikura S, Tominaga T. Efficacy of prophylactic blood pressure lowering according to a standardized postoperative management protocol to prevent symptomatic cerebral hyperperfusion after direct revascularization surgery for moyamoya disease. Cerebrovasc Dis. 2012;33(5): 436-45. 